\title{
Assessing submarine groundwater discharge dynamics using long-term high-resolution radon measurements and machine learning
}

\author{
Trista McKenzIE ${ }^{1 *}$, Henrietta Dulai ${ }^{1}$, Peter $^{2}$ \\ FULEKY $^{2}$, JONGHYUN LEE ${ }^{3}$ \\ ${ }^{1}$ Department of Earth Sciences, University of Hawai' $i$ at \\ Mānoa, Honolulu, HI 96822, USA (*correspondence: \\ tristam@hawaii.edu) \\ ${ }^{2}$ Department of Economics, University of Hawai'i at Mānoa, \\ Honolulu, HI 96822, USA \\ ${ }^{3}$ Department of Civil Engineering/Water Resources Research \\ Center, University of Hawai' $i$ at Mānoa, Honolulu, HI \\ 96822, USA
}

Submarine groundwater discharge (SGD) is an important contributor to coastal water and solute budgets [1]. Understanding coastal hydrology is increasingly important as climate change drives changes in hydrological and ocean parameters. Naturally-occurring radioisotopes such as ${ }^{222} \mathrm{Rn}$ (radon) are frequently used as tracers for SGD. This study analyzes a dataset collected by an autonomous gamma spectrometer [2] over a four-year deployment period that includes coastal radon activities, salinity, and water temperature off of the Kona coast of Hawai' $i$. Time-series data analysis were coupled with machine learning techniques with the objectives of (1) modeling and imputing missing radon concentrations using deep learning, (2) investigating SGD under future projections of sea level rise using the modeled SGD time-series, and (3) using machine learning algorithms such as random forest regression to explain the importance of the different terrestrial and marine drivers of SGD. Results to date demonstrated that the deep learning model accurately predicted the test data with mean square error of less than $2 \times 10^{-2}$. We also found that the tidal cycle is the primary driving force of SGD for this dataset. This research establishes the utility of combining field-based measurements with big-data driven approaches, in addition to offering insight into current and future SGD dynamics.

[1] Taniguchi et al. (2019) Front. Environ. Sci. 7, 1-26. [2] Dulai et al. (2016) J. Radioanal. Nucl. Chem. 307, 18651870 . 